\title{
Consistência das informações de um estudo sobre o impacto da assistência nutricional no atendimento pré-natal
}

\author{
Consistency of data in a study about the impact of nutritional assistance during prenatal care
}

\author{
Cláudia Saunders ${ }^{1}$, Patricia de Carvalho Padilha², Cristiane Barbosa Chagas ${ }^{3}$, Cristina Lúcia da Silva ${ }^{4}$, Elizabeth Accioly ${ }^{5}$, \\ Andréa Ramalho 6
}

\section{RESUMO}

Objetivo: Avaliar a consistência dos dados obtidos no estudo Avaliação do impacto da assistência nutricional pré-natal no resultado obstétrico, por análise da confiabilidade das informações e perdas de seguimento ocorridas.

Métodos: Trata-se de um estudo de intervenção, prospectivo, em uma coorte de gestantes. O grupo pré-intervenção (GI) foi constituído de 225 puérperas adultas (1999-2001) e o grupo intervenção (GII), de 208 gestantes adultas (20052006) e seus respectivos recém-nascidos, atendidos em Maternidade Pública do Rio de Janeiro. Visando à padronização dos procedimentos de coleta de dados, os pesquisadores receberam treinamento, reciclagem e foram supervisionados. Além disso, elaborou-se um manual de instruções para consulta no trabalho de campo, foi feito um estudo piloto para testar os instrumentos; a análise da consistência dos dados foi semanal. Para avaliar a confiabilidade da obtenção das informações, foram aplicadas as estatísticas Kappa (k) e o Índice de Concordância Intraclasse (ICC).

Resultados: As perdas de seguimento do GII foram de $8,4 \%$. Houve boa padronização dos procedimentos de obtenção das informações em ambos os grupos, com ICC $>0,92$ e k>0,65 no GI e, para o GII, ICC >0,94 e $\mathrm{k}>0,71$. Destaca-se a alta concordância entre o peso prégestacional informado e o aferido no primeiro trimestre (ICC $=0,961)$.

Conclusões: Os resultados sugerem que as estratégias adotadas no presente estudo contribuíram para o baixo percentual de perdas de seguimento e garantia da qualidade dos dados, permitindo a reprodutibilidade dos resultados.

Palavras-chave: estudos de intervenção; estudos de coorte; cuidado pré-natal.

\section{ABSTRACT}

Objective: To assess the consistency of the results obtained in the study Assessment of the impact of prenatal nutritional assistance on pregnancy outcome by testing the reliability of data and analyzing follow-up losses.

Methods: This is a prospective intervention study in a cohort of pregnant women. Pre-intervention group (GI) was formed by 225 postpartum women (1999-2001) and
Instituição: Instituto de Nutrição Josué de Castro da Universidade Federal do Rio de Janeiro (INJC/UFRJ); Rio de Janeiro, RJ, Brasil

${ }^{1}$ Nutricionista, Doutora em Ciências pela Escola Nacional de Saúde Pública (ENSP) da Fundação Oswaldo Cruz (Fiocruz), professor adjunto do Departamento de Nutrição e Dietética do INJC/UFRJ e da Maternidade Escola da UFRJ, coordenadora do Grupo de Pesquisa em Saúde Materna e Infantil (GPSMI) do Núcleo de Pesquisa em Micronutrientes (NPqM) do INJC/UFRJ, Rio de Janeiro, RJ, Brasil

${ }^{2}$ Nutricionista do Instituto de Puericultura e Pediatria Martagão Gesteira (IPPMG/UFRJ), doutoranda do Programa de Pós-graduação em Nutrição do INJC/UFRJ, pesquisadora do GPSMI/NPqM do INJC/UFRJ, Rio de Janeiro, RJ, Brasil

${ }^{3}$ Nutricionista do Programa Materno-Infantil do Departamento de Saúde Coletiva Itaguaí, Mestre em Nutrição pelo INJC/UFRJ, pesquisadora do GPSMI/NPqM do INJC/UFRJ, Rio de Janeiro, RJ, Brasil

${ }^{4}$ Nutricionista da Maternidade Escola/UFRJ, mestranda do Programa de Pós-graduação em Nutrição do INJC/UFRJ, pesquisadora do GPSMI/NPqM do INJC/UFRJ, Rio de Janeiro, RJ, Brasil

${ }^{5}$ Doutora em Ciências pela Universidade Federal de São Paulo, professor adjunto do Departamento de Nutrição e Dietética do INJC/UFRJ, docente pesquisadora do GPSMI/NPqM do INJC/UFRJ, Rio de Janeiro, RJ, Brasil
${ }^{6}$ Doutora em Ciências pela ENSP/Fiocruz, professora titular do Departamento de Nutrição Social e Aplicada, coordenadora do NPqM do INJC/UFRJ, Rio de Janeiro, RJ, Brasil

Endereço para correspondência:

Cláudia Saunders

Instituto de Nutrição Josué de Castro da UFRJ

Avenida Trompovsky, s/n, Edifício dos Institutos - Ilha do Fundão

Centro de Ciências da Saúde, Bloco J, $2^{\circ}$ andar, sala 26

CEP 21944-970 - Rio de Janeiro/RJ

E-mail: cfcoelho@osite.com.br/csaunders@ig.com.br

Fontes financiadoras: Auxílio financeiro do edital CNPq 51/2005, processo 402129/2005-9. Concessão de bolsas de Iniciação Científica da Fundação de Amparo à Pesquisa do Estado do Rio de Janeiro (Faperj), processos E-26/152.877/2004 E-26/152.876/2004

Recebido em: 25/6/08

Aprovado em: 2/10/08 
the intervention group (GII), by 208 pregnant women (2005-2006) and their respective newborn infants assisted in a public maternity hospital of Rio de Janeiro, Brazil. In order to standardize data collection, field researchers were trained, recycled and supervised, and an instruction guide was developed. A pilot study was done to test the tools and assessment of data consistency was performed weekly. Evaluation of reliability used Kappa (k) statistics and intraclass correlation coefficient (ICC).

Results: The follow-up losses of GII were $8.4 \%$. There was a good standardization of procedures to obtain information in both groups, with an ICC $>0.82$ and $\mathrm{k}>0.65$ for GI and ICC $>0.94$ and $k>0.71$ for GII. There was a high concordance between the pre-pregnancy weight informed and the weight measured in the first trimester (ICC $=0.96$ ).

Conclusions: The strategies adopted in the present study contributed to the low percentage of follow-up losses and to guarantee the quality of data, allowing reliability of results.

Key-words: intervention studies; cohort studies; prenatal care.

\section{Introdução}

No estudo da confiabilidade das informações, verifica-se a capacidade de obtenção de resultados com variações mínimas quando a pesquisa é realizada por diferentes pessoas ou em diferentes momentos ${ }^{(1,2)}$. A confiabilidade verificada em múltiplas avaliações do mesmo fenômeno é de vital importância nas investigações, constituindo um dos temas mais atuais da epidemiologia moderna ${ }^{(3,4)}$.

A sistematização da coleta de dados, com passos de conduta definidos e ordenados, tende a melhorar a qualidade e a reprodutibilidade das informações coletadas. Há diversas maneiras de verificar a concordância de resultados entre leituras de um mesmo evento e, assim, estimar o erro cometido em sua aferição. O tipo de dado coletado, que gerará a construção das variáveis consideradas em um estudo (categóricas, contínuas, discretas ou ordinais), influencia a forma de análise dos dados. Assim, para cada tipo de variável, a estatística deve ser selecionada e aplicada corretamente para que se verifique o índice de concordância entre os entrevistadores e, dessa forma, seja evidenciada a desejável existência de padronização nos procedimentos de obtenção de dados confiáveis ${ }^{(2,3,5-7)}$.
Outro aspecto a ser considerado por pesquisadores é a avaliação das perdas de informações dos integrantes do estudo e, no caso de estudos de coorte, nos quais os indivíduos serão acompanhados por um período variável, dependendo do objetivo do estudo, as perdas de seguimento devem ser previstas e analisadas. Ao longo do estudo, é preciso empregar estratégias para minimizar as perdas e motivar os integrantes a participarem até o fim do período previsto.

O presente estudo tem como objetivo avaliar a consistência das informações obtidas no estudo Avaliação do impacto da assistência nutricional pré-natal no resultado obstétrico por análise da confiabilidade das informações obtidas e das perdas de seguimento ocorridas.

\section{Métodos}

Este artigo foi escrito com base nos dados da pesquisa Avaliação do impacto da assistência nutricional pré-natal no resultado obstétrico, cujo objetivo foi esclarecer a relação de causa e efeito da intervenção nutricional no desfecho obstétrico ${ }^{(2,8)}$.

Foram definidos dois grupos de estudo: o Grupo Pré-Intervenção (GI) e o Grupo Intervenção (GII). O GI foi constituído por mulheres nas primeiras seis horas após o parto e seus respectivos recém-nascidos internados na Maternidade Escola da UFRJ no período de abril de 1999 a dezembro de 2001. Dentre 252 puérperas, selecionaram-se as 225 mulheres adultas de acordo com os seguintes critérios de inclusão: gestantes de feto único, não-portadoras de enfermidades clinicamente comprovadas com início no período do pré-gestacional (diabetes melito, hepatopatias, cardiopatias e outras) e não-usuárias de suplemento vitamínico-mineral contendo vitamina A no período gestacional. Esse grupo foi considerado um controle histórico. O GII foi constituído por 227 gestantes acompanhadas no ambulatório de assistência pré-natal da Maternidade Escola da Universidade Federal do Rio de Janeiro (UFRJ). Todas as gestantes presentes na unidade no período de junho de 2005 a janeiro de 2006 e que atenderam aos critérios de inclusão (idade cronológica $\geq 20$ anos, início da assistência pré-natal até a $16^{a}$ semana gestacional, gestação de feto único, não-portadoras de enfermidades com início prévio à gestação) foram convidadas a integrar o estudo. A intervenção nutricional foi elaborada com base em avaliação nutricional detalhada e orientação individualizada. As gestantes/puérperas e os recémnascidos que constituíram o GII foram acompanhados até o puerpério imediato, quando se avaliou o impacto da assistência nutricional pré-natal nos desfechos de interesse. 
A equipe de trabalho foi composta por alunos de graduação, aperfeiçoandos, mestrandos, doutorandos, nutricionistas, supervisor/coordenador de campo e pela coordenadora geral do estudo, todos vinculados ao Grupo de Pesquisa em Saúde Materna e Infantil/Núcleo de Pesquisa em Micronutrientes (NPqM) do Instituto de Nutrição Josué de Castro (INJC) e ao Serviço de Nutrição da Maternidade Escola, ambos da UFRJ.

$\mathrm{O}$ treinamento da equipe teve carga horária de 40 horas e duas etapas. A primeira etapa contou com a participação dos pesquisadores do NPqM do INJC/UFRJ, com experiência acumulada em pesquisa e trabalhos de campo, discussão sobre as estratégias de captação da amostra, aplicação dos questionários e discussão de medidas para reduzir as perdas de seguimento. A segunda etapa, com a participação de toda a equipe de campo, foi dividida em dois momentos: primeiramente foi feita uma ambientação à unidade do estudo, discussão sobre ética em pesquisa, etapas do estudo, instrumento de coleta de dados e terminologias técnicas empregadas na área da saúde. A seguir, realizou-se o treinamento para o preenchimento dos instrumentos, com simulação de aplicação, autoavaliação pelos pesquisadores da suficiência das respostas obtidas, discussão sobre as técnicas de abordagem e captação das gestantes.

Ainda no treinamento, discutiu-se o contexto do aconselhamento nutricional e desenvolveu-se um modelo a ser seguido durante as consultas com o nutricionista, sendo que técnicas foram implementadas com o objetivo de contribuir para o aperfeiçoamento da equipe na assistência às gestantes, estabelecer um vínculo com elas e minimizar as perdas de seguimento. Tal modelo foi estruturado com base na literatura disponível ${ }^{(9)}$ e na experiência acumulada na prática em obstetrícia. Dessa forma, foram usadas habilidades específicas - escuta atenciosa, criação do vínculo por meio da empatia, estabelecimento do diálogo, encorajamento, elogio e aplicação da sugestão em vez da imposição, com o propósito de aumentar a adesão das gestantes ao cuidado nutricional proposto. Medidas simples, como chamar a gestante pelo nome, perguntar sobre o concepto, referindo-se a ele pelo nome, e o atendimento pelo mesmo profissional nas consultas de retorno, também foram observadas.

Todos os pesquisadores foram orientados a seguir o manual de instruções detalhado para consultas no trabalho de campo. Houve, ainda, a elaboração de um livro de registro de ocorrências no trabalho de campo e as dúvidas eram resolvidas pelo supervisor de campo ou pelos coordenadores. A supervisão de campo foi realizada por nutricionistas aperfeiçoandas e a coordenação de campo foi realizada por mestrandos com experiência em pesquisa. Ambos tinham as atribuições de elaborar escalas de trabalho e distribuir tarefas, verificar a suficiência de material de trabalho na coleta de dados e manter contato permanente com a coordenadora geral do estudo. Garantiu-se, também, um horário semanal de reuniões científicas para atualização da equipe.

O piloto ocorreu na Maternidade Escola da UFRJ para o estudo com o GI em 1999 ( $\mathrm{n}=35 ; 13,4 \%$ da amostra) e para o GII em 2005 ( $n=28 ; 12,3 \%$ da amostra). Os instrumentos foram testados, ajustados e os dados coletados então não foram incorporados à amostra final do estudo.

A análise da consistência das informações foi realizada semanalmente pelos coordenadores. Realizou-se a revisão dos questionários preenchidos, com avaliação da suficiência das informações e identificação de dados incompletos ou duvidosos, resolvidos por meio de reconsulta aos prontuários. Com base nessa análise, periodicamente foi realizada a reciclagem da equipe, visando à obtenção de dados seguros e fidedignos.

Antes da digitação, foram feitos os cálculos necessários para a estimativa de variáveis contínuas (como, por exemplo, renda familiar total e/ou per capita, ganho de peso gestacional total, índice de massa corpórea, IMC) e verificação de outliers (valores improváveis). A digitação foi realizada pelo coordenador de campo, com supervisão da coordenadora geral.

Para analisar a qualidade dos dados, realizou-se a avaliação da confiabilidade de aplicação, medindo-se a equivalência dos resultados obtidos com a aplicação de um mesmo instrumento por entrevistadores diferentes, para um mesmo paciente integrante do estudo ${ }^{(1)}$. Para avaliar a concordância interobservador, foram selecionadas variáveis contínuas e categóricas referentes aos dois grupos de estudo (GI e GII).

No GI, dois diferentes entrevistadores coletaram, de forma independente, as informações referentes à puérpera, por meio de consulta aos prontuários e entrevista com 33 (12,6\% do total da amostra final) integrantes do estudo selecionadas aleatoriamente ainda no período de internação na unidade estudada. No GII, dois entrevistadores coletaram informações, também de forma independente, dos prontuários de $11 \%$ das gestantes $(n=25)$ integrantes do estudo. A seleção dos prontuários para a coleta de dados ocorreu aleatoriamente, dentre aqueles que haviam sido preenchidos pelo coordenador do trabalho de campo no atendimento à gestante.

Consideraram-se como 'perdas', os casos nos quais não foi possível obter informações referentes aos desfechos - adequação do ganho de peso gestacional total, peso ao nascer e/ou intercorrências maternas e do recém-nascido.

$\mathrm{Na}$ análise dos dados, em avaliação exploratória, a identificação e a exclusão dos outliers (valores extremos), 
para as variáveis contínuas, foram definidas para valores acima ou abaixo de menos três desvios padrão da média de tal valor ${ }^{(10)}$.

Calcularam-se as medidas de tendência central e de dispersão das variáveis contínuas. O teste de MannWhitney foi empregado para testar a igualdade de duas médias. $\mathrm{Na}$ análise bivariada, verificou-se a associação entre as variáveis dependentes (desfecho) e as independentes por meio do teste do qui-quadrado. O nível de significância estatística adotado foi de $5 \%$. As análises foram feitas no pacote estatístico SPSS, versão 13.0.

Empregou-se a estatística Kappa (k) para avaliar a reprodutibilidade das variáveis categóricas ${ }^{(2,11)}$ e definiu-se o intervalo de confiança (IC) de $95 \%{ }^{(12)}$. O coeficiente de correlação intraclasse (ICC), com IC de 95\%, foi calculado para avaliar a concordância de variáveis contínuas ou ordinais ${ }^{(2,11)}$. $\mathrm{Na}$ interpretação dos resultados, adotou-se a classificação proposta por Landis e Koch ${ }^{(13)}$, considerando-se índices >0,61 como boa concordância.

A amostra final do estudo foi constituída por 262 puérperas incluídas no GI (225 adultas e 37 adolescentes) e seus recém-nascidos. Estes foram atendidos na Maternidade Escola da UFRJ, antes da implantação da intervenção. Diante do perfil de saúde e nutrição desse grupo, procedeu-se à revisão das rotinas de atendimento

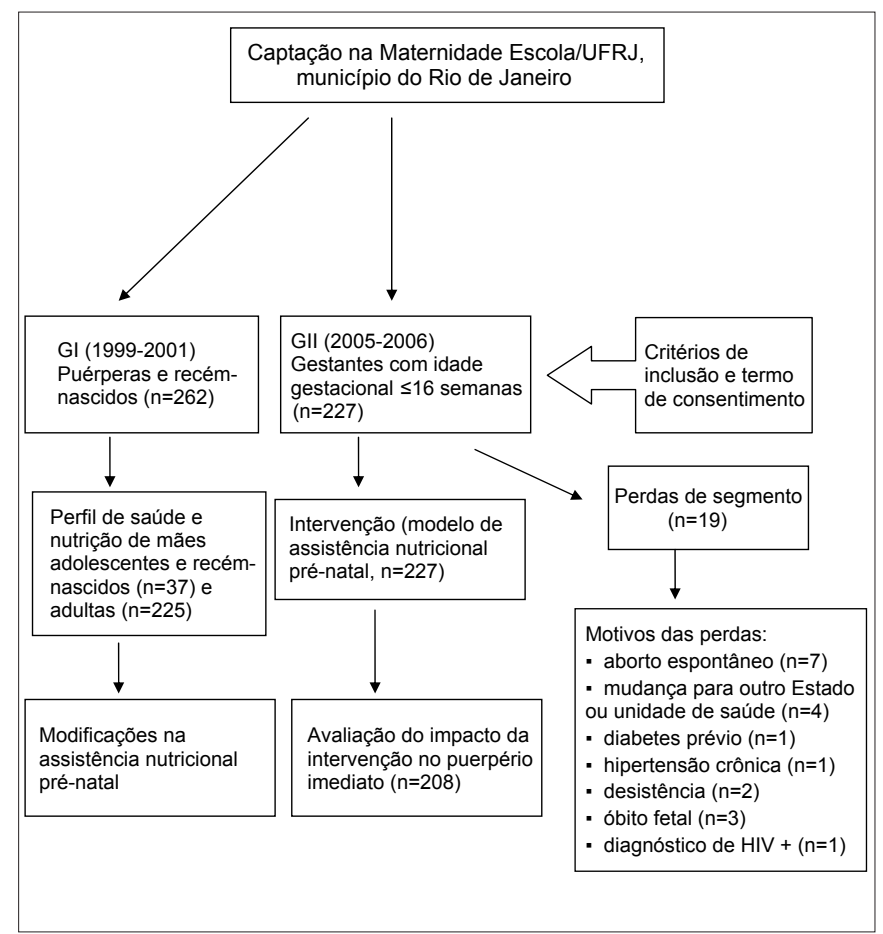

Figura 1 - Representação do processo de seleção dos grupos de estudo, coleta de dados e perdas de seguimento. nutricional da unidade e elaborou-se uma proposta de assistência nutricional pré-natal, com a previsão de um calendário mínimo de consultas com o nutricionista, iniciado de modo concomitante ao da assistência prénatal da unidade (Figura 1). O GII foi constituído por 227 gestantes adultas atendidas na Maternidade Escola da UFRJ incluídas na coorte até a $16^{a}$ semana de gestação e que receberam a intervenção (proposta de assistência nutricional pré-natal). Na Figura 1 é descrito o processo de captação da amostra dos GI e GII, a coleta de dados e são apresentadas as perdas de seguimento. $\mathrm{Na}$ análise exploratória dos dados, foram excluídos os outliers para as seguintes variáveis: ganho de peso gestacional total $(n=6)$ e número de gestações $(n=7)$.

O estudo obedeceu aos princípios éticos ${ }^{(14)}$ e foi aprovado pelo Comitê de Ética em Pesquisa do Instituto de Puericultura e Pediatria Martagão Gesteira.

\section{Resultados}

Analisando-se as perdas de seguimento do GII, verifica-se que foi de $8,4 \%$ ( $n=19$, Figura 1$)$, percentual inferior ao estimado. $\mathrm{Na}$ investigação de diferenças entre as mulheres incluídas no estudo e as definidas como perdas, verifica-se similaridade entre ambas: idade materna $(p=0,73)$; renda familiar per capita $(p=0,62)$; número de gestações $(p=0,33)$; número de abortos ( $p=0,83)$; IMC pré-gestacional $(p=0,45)$; situação marital $(p=0,95)$; cor da pele $(p=0,55)$; condições de saneamento da moradia $(p=0,61)$; classificação do IMC pré-gestacional $(p=0,24)$. Identificou-se uma maior proporção de mulheres com melhor grau de escolaridade no grupo de perdas $(p=0,02)$.

Os índices de concordância entre os pesquisadores que participaram do estudo na obtenção das informações do GI indicam boa padronização dos procedimentos de obtenção das informações, a julgar pelos valores de ICC $(>0,92)$ e $\mathrm{k}(>0,65)$ obtidos. O menor índice de $\mathrm{k}$ foi identificado para a variável intercorrências gestacionais (Tabela 1).

Quanto aos índices de concordância entre os pesquisadores que participaram do estudo com o GII, verificou-se também boa padronização na obtenção das informações, com índices de ICC >0,94 e k>0,71. A variável de menor índice de k foi gestação planejada (Tabela 2).

Finalmente, houve concordância entre o peso pré-gestacional informado pela gestante, referente ao período de até dois meses antes da concepção, e o peso aferido até o final da $13^{\mathrm{a}}$ semana gestacional $($ ICC $=0,96$; IC95\% $=0,94-0,98)$. 
Tabela 1 - Comparação entre as informações obtidas por diferentes entrevistadores para o $G$ I $(n=33)$.

\begin{tabular}{|c|c|c|}
\hline \multirow[b]{2}{*}{ Variáveis contínuas ou ordinais } & \multicolumn{2}{|c|}{ Concordância } \\
\hline & ICC & IC95\% \\
\hline Idade materna (anos) & 0,99 & $0,998-0,999$ \\
\hline Número de gestações & 1,00 & - \\
\hline Número de abortos & 1,00 & - \\
\hline Idade gestacional (DUM) no parto (semanas) & 0,96 & $0,917-0,979$ \\
\hline Peso pré-gestacional (kg) & 0,99 & $0,977-0,994$ \\
\hline Estatura (m) & 0,98 & $0,949-0,987$ \\
\hline Peso pré-parto (kg) & 0,96 & $0,925-0,982$ \\
\hline Peso ao nascer $(\mathrm{kg})$ & 0,97 & $0,944-0,986$ \\
\hline Comprimento ao nascer $(\mathrm{cm})$ & 0,99 & $0,991-0,998$ \\
\hline Condições de saneamento da moradia (adequada/inadequada) & 1,00 & - \\
\hline Intercorrências gestacionais (sim/não) & 0,65 & $0,390-0,910$ \\
\hline Cegueira noturna gestacional (sim/não) & 1,00 & - \\
\hline
\end{tabular}

ICC: coeficiente de correlação intraclasse; k: estatística Kappa; IC: intervalo de confiança.

Tabela 2 - Comparação entre as informações obtidas por diferentes entrevistadores para o GII $(n=25)$.

\begin{tabular}{lcc}
\hline & \multicolumn{2}{c}{ Concordância } \\
\hline Variáveis contínuas ou ordinais & ICC & IC95\% \\
\hline Idade maternal (anos) & 0,99 & $0,9947-0,9990$ \\
Número de gestações & 1,00 & - \\
Renda familiar total (salários mínimos) & 1,00 & - \\
Idade gestacional (DUM) 1a consulta (semanas) & 0,97 & $0,9234-0,9858$ \\
Peso pré-gestacional (kg) & 0,94 & $0,8699-0,9753$ \\
Estatura (m) & 0,99 & $0,9995-0,9999$ \\
Ganho de peso total (kg) & 0,95 & $0,8631-0,9784$ \\
VET (Kcal) & 1,00 & - \\
Hemoglobina (g/dL) & 1,00 & - \\
Glicemia (mg/dL) & 0,96 & $0,8883-0,9846$ \\
IMC pré-gestacional (kg/m²) & 0,95 & $0,8963-0,9797$ \\
IMC gestacional (kg/m²) & 0,99 & $0,9996-1,0000$ \\
Pressão arterial (mmHg) & 1,00 & - \\
\hline Variáveis categóricas & $\mathbf{k}$ & $\mathbf{I C 9 5 \%}$ \\
\hline Estado civil (casada ou vive com companheiro/vive sem companheiro) & 1,00 & - \\
Escolaridade (analfabeta/ensino fundamental incompleto/ensino fundamental completo/ & & - \\
ensino médio incompleto/ensino médio completo/superior) & 1,00 & - \\
Condições de saneamento da moradia (adequado/inadequado) & 1,00 & - \\
Cegueira noturna gestacional (sim/não) & 1,00 & - \\
Gestação foi planejada? (sim/não) & 0,71 & $0,36-1,00$ \\
Edema (sim/não) & 1,00 & - \\
Intercorrências gestacionais (sim/não) & 1,00 & $0,29-0,89$ \\
Sintomatologia digestiva (sim/não) & 0,80 & $0,54-1,00$ \\
IMC pré-gestacional (baixo peso, normal, sobrepeso, obesidade) & 0,92 & $0,78-1,00$ \\
IMC gestacional (baixo peso, adequado, sobrepeso, obesidade) & 1,00 & $0,50-1,00$ \\
\hline
\end{tabular}

ICC: coeficiente de correlação intraclasse; k: estatística Kappa; IC: intervalo de confiança. 


\section{Discussão}

No presente estudo, os cuidados empregados na tentativa de melhorar a qualidade das informações foram indispensáveis para obter dados confiáveis, tais como: realização de treinamento teórico-prático da equipe envolvida na coleta de dados, reciclagem periódica, supervisão constante, verificação do preenchimento dos formulários da pesquisa, manutenção de equipe fixa, integração entre os pesquisadores e elaboração de manual de instruções para o preenchimento correto do formulário, treinamento, reciclagem, além da supervisão constante.

Trata-se de estudo de seguimento, que pode ser invalidado caso as perdas sejam grandes, da ordem ou superior a 30\%. No presente estudo, as perdas foram de $8,4 \%$, proporção inferior à estimada. Vale ressaltar que, das 19 perdas de seguimento, apenas duas ocorreram por desistência. Tal achado pode estar relacionado à concretização da prática do aconselhamento nutricional no desenvolvimento de um relacionamento estreito, de confiança e de longo prazo entre o profissional de saúde e a gestante $^{(15)}$. As perdas de seguimento podem comprometer a validade dos resultados e, se há grande quantidade de perdas, as estimativas de associação geradas por tais dados podem não refletir os achados de forma fidedigna ${ }^{(16)}$.

Recentemente, em um estudo de coorte sobre os determinantes do ganho ponderal excessivo em gestantes de baixo risco atendidas em serviço público de pré-natal, houve perda de $37,8 \%$ dentre as elegíveis para o estudo ${ }^{(17)}$. As causas das perdas mencionadas pelos autores foram: impossibilidade de obtenção de informação quanto ao peso ao nascer, abandono do pré-natal, mudança de cidade e recusas.

Araújo et al ${ }^{(16)}$, em estudo de revisão sistemática sobre ansiedade, prematuridade e baixo peso, consideraram, dentre os eixos para análise da qualidade dos estudos, as perdas de seguimento. Dos 13 estudos selecionados para a referida revisão, 11 foram de coorte, um caso-controle e um transversal, sendo as informações sobre as perdas de seguimento obtidas em nove dos 13 estudos. As perdas variaram entre $10,3^{(18)}$ a $36,9 \%{ }^{(19)}$.

As medidas empregadas ao longo do presente estudo para minimizar essas perdas foram: busca ativa das gestantes na sala de espera da unidade, localização das integrantes do estudo que tiveram seus filhos em outras unidades de saúde, atualização constante do endereço e telefone das gestantes, contato telefônico com as faltosas e marcação de novas datas para as consultas, destacando-se, especialmente, as técnicas de aconselhamento nutricional empregadas, que contribuíram para a melhoria do acolhimento e para a criação do vínculo com o profissional.

No estudo da reprodutibilidade das informações coletadas, verificaram-se bons índices de concordância entre os entrevistadores para as variáveis avaliadas, evidenciando-se que a padronização nos procedimentos de obtenção das informações foi adequada. No estudo do GI, verificou-se maior dificuldade dos pesquisadores para identificar as intercorrências gestacionais. Com isso, na reciclagem dos pesquisadores, reforçaram-se as informações sobre a busca de sinais e alterações nos exames de rotina associados às intercorrências, além da localização e interpretação do parecer médico nos prontuários. A dificuldade de detectar as intercorrências gestacionais pode decorrer de problemas do prontuário, pois a intercorrência pode não ter sido anotada ou pode ter ocorrido falta de normatização do conteúdo a ser descrito no prontuário(3).

No estudo do GII, a variável de menor índice de concordância foi 'gestação planejada'. Tal informação é investigada por meio de consulta aos pareceres dos profissionais do serviço social ou de psicologia na unidade. Assim, na reciclagem dos pesquisadores, reforçou-se a necessidade de consulta detalhada aos pareceres dos referidos profissionais.

Diante da concordância observada entre o peso informado pelas gestantes, correspondente a no máximo dois meses antes da concepção, com aquele medido ainda no primeiro trimestre, sugere-se que ambos podem ser utilizados para o cálculo do IMC pré-gestacional ${ }^{(20)}$. Contudo, é preferível que o peso informado pela gestante seja adotado na sua avaliação inicial, por não considerar o ganho de peso gestacional na estimativa do IMC inicial, pois, embora o ganho de peso materno no primeiro trimestre seja menor em comparação aos outros trimestres ${ }^{(21,22)}$, este ainda ocorre, podendo variar teoricamente de 0,9 a $2,3 \mathrm{~kg}^{(21)}$.

Pode-se concluir que o baixo percentual de perdas de seguimento da coorte reflete a eficácia das estratégias adotadas para minimizar as perdas e melhorar a qualidade dos dados obtidos, repercutindo no maior comparecimento das gestantes às consultas com o nutricionista. Dessa forma, é necessário enfatizar a importância de planejamento criterioso e do emprego de estratégias adequadas que garantam a qualidade das informações coletadas para pesquisas, objetivando sua reprodutibilidade e extrapolação dos resultados, tendo em vista que a qualidade da informação é essencial para subsidiar o planejamento e a avaliação de ações e programas de saúde. 


\section{Referências bibliográficas}

1. Almeida Filho N, Rouquayrol MZ. Diagnóstico em Epidemiologia. In: Rouquayrol MZ, Almeida Filho N, editors. Introdução à epidemiologia. $4^{\mathrm{a}}$ ed. Rio de Janeiro: Guanabara Koogan; 2006. p. 86-106.

2. Pereira MG. Aferição dos eventos. In: Pereira MG. Epidemiologia: teoria e prática. Rio de Janeiro: Guanabara Koogan; 1995. p. 358-76.

3. Campos MR, Leal MC, Cunha CB, Souza Jr PR. Consistency between data sources and inter-observer reliability in the study on neonatal and perinatal morbidity and mortality and care in the City of Rio de Janeiro. Cad Saude Publica 2004;20(Suppl 1):S34-43.

4. Griep RH, Chor D, Faerstein E, Lopes C. Social support: scale test-retest reliability in the Pro-Health Study. Cad Saude Publica 2003;19:625-34.

5. Shrout $\mathrm{PE}$, Fleiss $\mathrm{JL}$. Intraclass correlations: uses in assessing rater reliability. Psychol Bull 1979;86:420-8.

6. Fleiss JL. Determining sample sizes needed to detect a difference between two proportions. In: Fleiss JL; Levin B; Paik MC, editors. Statistical methods for rates and proportions. New York: John Wiley; 1981. p. 33-49.

7. Cohen J. A coefficient of agreement for nominal scales. Educ Psychol Meas 1960;20:37-46

8. Escosteguy CC. Estudos de intervenção. In: Medronho RA, Carvalho DM, Bloch KV, Luiz RR, Werneck GL, editors. Epidemiologia: teoria e prática. São Paulo: Atheneu; 2002. p. 151-60.

9. Rodrigues EM, Soares FP, Boog MC. Resgate do conceito de aconselhamento no contexto do atendimento nutricional. Rev Nutr 2005;18:119-28.

10. Hair JF, Anderson RE, Tatham RL, William CB. Examining your data. In: Multivariate data analysis: with readings. $4^{a}$ ed. New Jersey: Prentice Hall; 1995. p. 32-75.

11. Szklo M, Nieto FJ. Quality assurance and control. In: Szklo M, Nieto FJ, editors. Epidemiology: beyond the basics. Maryland: Aspen; 2000. p. 343-404.
12. Abramson JH, Gahlinger PM. Computer programs for epidemiologists (PEPI). Kappa-Agreement between categorical ratings. Version 3.0. Stone Mountain: USA; 1993-1999.

13. Landis JR, Koch GG. The measurement of observer agreement for categorica data. Biometrics 1977;33:159-74.

14. Brasil - Ministério da Saúde. Diretrizes e normas regulamentadoras de pesquisas envolvendo seres humanos. Resolução 196/96 do Conselho Nacional de Saúde. Rio de Janeiro: Fundação Oswaldo Cruz, 1998.

15. Saunders C, Santana M. Avaliação do impacto da assistência nutricional pré-natal no resultado obstétrico [relatório final: processo E-26/152.877/2004 - Faperj]. Rio de Janeiro: UFRJ; 2006.

16. Araújo DM, Pereira NL, Kac G. Anxiety during pregnancy, prematurity, and low birth weight: a systematic literature review. Cad Saude Publica 2007;23:747-56.

17. Stulbach TE, Benício MH, Andreazza R, Kono S. Determinants of excessive weight gain during pregnancy in a public low risk antenatal care service. Rev Bras Epidemiol 2007;10:99-108.

18. Burstein I, Kinch RA, Stern L. Anxiety, pregnancy, labor, and the neonate. Am J Obstet Gynecol 1974;118:195-9.

19. Mancuso RA, Schetter CD, Rini CM, Roesch SC, Hobel CJ. Maternal prenatal anxiety and corticotropin-releasing hormone associated with timing of delivery. Psychosom Med 2004;66:762-9.

20. World Health Organization. Physical Status: the use and interpretation of anthropometry. WHO Technical Report Series $n^{\circ}$ 854. Geneva, Switzerland: WHO, 1995

21. Institute of Medicine. Nutrition during pregnancy. Washington DC: National Academy Press; 1990.

22. Brasil - Ministério da Saúde. Pré-natal e puerpério: atenção qualificada e humanizada. Brasília: Ministério da Saúde; 2006. 